\title{
Lidil
}

Revue de linguistique et de didactique des langues

$62 \mid 2020$

Recherches actuelles en didactique du lexique: avancées, réflexions, méthodes

\section{Maurice Niwese, Jacqueline Lafont-Terranova et Martine Jaubert (dir.), Écrire et faire écrire dans l'enseignement postobligatoire. Enjeux, modèles et pratiques innovantes}

Villeneuve-d'Ascq, Presses universitaires du Septentrion, 2019, 400 p.

Luca Pallanti

\section{(2) OpenEdition}

Édition électronique

URL : http://journals.openedition.org/lidil/8221

DOI : $10.4000 /$ lidil.8221

ISSN : 1960-6052

Éditeur

UGA Éditions/Université Grenoble Alpes

\section{Édition imprimée}

ISBN : 978-2-37747-226-0

ISSN : $1146-6480$

Référence électronique

Luca Pallanti, « Maurice Niwese, Jacqueline Lafont-Terranova et Martine Jaubert (dir.), Écrire et faire écrire dans l'enseignement postobligatoire. Enjeux, modèles et pratiques innovantes », Lidil [En ligne], 62 | 2020, mis en ligne le 03 novembre 2020, consulté le 10 décembre 2020. URL : http:// journals.openedition.org/lidil/8221 ; DOI : https://doi.org/10.4000/lidil.8221

Ce document a été généré automatiquement le 10 décembre 2020.

(c) Lidil 


\section{Maurice Niwese, Jacqueline Lafont- Terranova et Martine Jaubert (dir.), Écrire et faire écrire dans l'enseignement postobligatoire. Enjeux, modèles et pratiques innovantes}

Villeneuve-d'Ascq, Presses universitaires du Septentrion, 2019, 400 p.

Luca Pallanti

\section{RÉFÉRENCE}

Maurice Niwese, Jacqueline Lafont-Terranova et Martine Jaubert (dir.), Écrire et faire écrire dans l'enseignement postobligatoire. Enjeux, modèles et pratiques innovantes, Villeneuve-d'Ascq, Presses universitaires du Septentrion, 2019, $400 \mathrm{p}$.

1 Avec une parution en 2019, l'ouvrage Écrire et faire écrire dans l'enseignement postobligatoire. Enjeux, modèles et pratiques innovantes conclut une décennie féconde pour les recherches sur la formation à l'écriture. Les trois parties composant le volume, dirigé par M. Niwese, J. Lafont-Terranova et M. Jaubert, s'intéressent aux pratiques d'écriture "à l'intersection entre le monde universitaire et le monde du travail » (p. 12). On y questionne d'abord les modèles théoriques en didactique de l'écriture, puis la formation des futurs enseignants et, in fine, les dispositifs didactiques innovants. Ce sont les enjeux liés à l'enseignement/apprentissage de l'écrit, abordés sous un angle sociocognitif, qui constituent le liant des 18 articles composant l'ouvrage.

2 Si la préface de S.Plane a le mérite d'introduire le lecteur aux littéracies postsecondaires, le chapitre conclusif, signé par C.Blaser, D. Colin, S. Colognesi et R. Gagnon propose une comparaison utile des systèmes de formation à l'enseignement de l'écriture en francophonie. Entre ces deux repères, les trois parties évoluent dans 
une progression cohérente où l'on défend l'innovation didactique, entendue comme la capacité à « imaginer des dispositifs qui bousculent les frontières entre les didactiques $[\ldots] »($ p. 340).

3 Les 6 articles regroupés dans la première partie de l'ouvrage font office de chapeau théorique pour les parties qui suivent. On y aborde, notamment, le rapport métacognitif à l'écriture de recherche en contexte universitaire, envisagée comme un moyen d'acquisition d'une posture réflexive et distanciée, en vue de l'insertion dans le monde professionnel. E. Lang, M. Makassikis, J.-P. Meyer et J.-C. Pellat étudient les processus structurant l'activité d'écriture à travers une comparaison des écrits d'étudiants natifs et allophones. Se former au raisonnement par le biais de techniques de rédaction diverses, comme le couplage inventio-argumentation dans l'article de M. Monte, ou les discours des rédacteurs experts en communication dans la contribution de C.Beaudet et V.Rey, constituent autant de pistes didactiques prometteuses. Dans la même lignée, l'étude du degré de conscience des étudiants face à leurs propres compétences scripturales menée par F. Boch, J. Sorba et P. Bessonneau montre «l'intérêt de travailler sur ce facteur métacognitif car il donne des espoirs d'efficacité de remédiation et d'entrainement » (p. 88). Attention, cependant, aux partis pris. Les résultats des entretiens réalisés par $\mathrm{K}$. Bellegarde révèlent à quel point des préjugés sédimentés de l'écrit " indiquent une résistance au processus d'acculturation » à l'écriture (p. 120). Enfin, dans une perspective critique, C. Morinet rappelle les biais conceptuels du scriptisme: travailler l'écrit sans tenir compte de l'oral desservirait la formation à l'écriture même.

4 Véritable pivot conceptuel de la deuxième partie du volume, l'article de M.-L. Elalouf analyse les avantages en recherche et en formation dérivants de l'exploitation des corpus scolaires. Versant recherche, les études de M. Niwese et P. Schneeberger, de M. Jaubert et Y. Lhoste, et de S. Doussot, illustrent comment l'analyse outillée des indices polyphoniques, des ruptures discursives ou encore des modes d'appropriation du discours d'autrui mettent en évidence les difficultés des étudiants à s'approprier les écrits scientifiques à forte dominante dialogique, tant du point de vue de la construction du sens que du processus scriptural. Dans le même sillage, l'originale contribution de C. Dolignier met en relation les impasses cognitives dans l'écriture plagiaire avec les «difficultés de l'exogénèse dans l'écriture scientifique» (p.176). Enfin, versant formation, l'article de V. Paolacci et N. Rossi-Gensane et celui de A. Saoussany montrent à quel point les étudiants en formation à visée professionnalisante nécessitent un entrainement aux mécanismes d'autorévision, qu'il s'agisse de l'emploi de la virgule, des procédés de segmentation au niveau micro et macro-syntaxique et, en général, de la gestion de la connexité.

5 La conception et l'expérimentation de dispositifs d'enseignement dits innovants sont au centre de la réflexion de la troisième partie du volume. Quatre articles sur cinq se proposent d'analyser les effets de l'atelier d'écriture créative. A.-M. Petitjean compare le modèle étatsunien de l'atelier d'écriture avec l'atelier à la française et met en avant «un élargissement de l'empan critique» (p.267) caractérisant ce dispositif, ce qui laisse apercevoir des développements majeurs dans les années à venir. L'article de J. Lafont-Terranova, qui s'inscrit en milieu francophone, et les études de C. Dompmartin-Normand, et de M. Cros et N. Mathis, centrées sur l'atelier d'écriture en classe de FLE, montrent en quoi cet anti-modèle scolaire se fondant sur la valorisation du geste créatif permet de dépasser les blocages psychologiques liés aux tâches 
d'écriture. Axée sur les savoirs objectivés effectivement enseignés, la contribution de M. Surian creuse, quant à elle, les pratiques d'enseignement en classe d'accueil postobligatoire à Lausanne. L'analyse des séquences révèle que l'enseignement des aspects textuels se manifeste à travers un entrainement quasi systématique des opérations de planification et de révision, ce qui permet, par ailleurs, une approche pragmatique et intégrative de l'enseignement de la langue.

6 En conclusion, la convergence des résultats des différentes études montre que la didactique des compétences rédactionnelles a atteint un niveau de maturité pendant la décennie 2010-2020. On aperçoit ainsi l'aube d'une ère nouvelle de la formation à l'écriture dans l'enseignement postobligatoire, qui reste, à bien des égards, un vaste domaine encore à explorer.

\section{AUTEURS}

LUCA PALLANTI

LIDILEM (EA 609), Université Grenoble Alpes 